\title{
Proteinlike dynamical transition of hydrated polymer chains
}

\author{
L. Tavagnacco $\odot,{ }^{1}$ M. Zanatta $\odot,{ }^{2}$ E. Buratti $\odot,{ }^{1}$ B. Rosi $\odot,{ }^{3}$ B. Frick, ${ }^{4}$ F. Natali $\odot,{ }^{5}$ J. Ollivier, ${ }^{4}$ E. Chiessi $\odot,{ }^{6}$ M. Bertoldo, ${ }^{7,8}$ \\ E. Zaccarelli ${ }^{\circ},{ }^{1, *}$ and A. Orecchini ${ }^{3}{ }^{3,9, \dagger}$ \\ ${ }^{1}$ ISC, CNR and Department of Physics, Sapienza University of Rome, 00185 Roma, Italy \\ ${ }^{2}$ Department of Physics, University of Trento, 38123 Trento, Italy \\ ${ }^{3}$ Department of Physics and Geology, University of Perugia, 06123 Perugia, Italy \\ ${ }^{4}$ Institut Laue Langevin, 38042 Grenoble, France \\ ${ }^{5}$ Operative Group Grenoble, IOM, CNR, Institut Laue Langevin, 38042 Grenoble, France \\ ${ }^{6}$ Department of Chemical Science and Technologies, University of Rome Tor Vergata, 00133 Roma, Italy \\ ${ }^{7}$ Department of Chemical and Pharmaceutical Sciences, University of Ferrara, 44121 Ferrara, Italy \\ ${ }^{8}$ ISOF, CNR, 40129 Bologna, Italy \\ ${ }^{9}$ IOM, CNR, c/o Department of Physics and Geology, University of Perugia, 06123 Perugia, Italy
}

(Received 17 July 2020; revised 30 October 2020; accepted 24 December 2020; published 26 February 2021)

\begin{abstract}
Combining elastic incoherent neutron scattering experiments at different resolutions with molecular dynamics simulations, we report the observation of a proteinlike dynamical transition in linear chains of $\operatorname{poly}(N$ isopropylacrylamide). We identify the onset of the transition at a temperature $T_{d}$ of about $225 \mathrm{~K}$. Due to a global fit procedure, we find quantitative agreement between measured and calculated polymer mean-squared displacements at all temperatures and time resolutions. Our results confirm the generality of the dynamical transition in macromolecular systems in aqueous environments, independently of the internal polymer topology.
\end{abstract}

DOI: 10.1103/PhysRevResearch.3.013191

\section{INTRODUCTION}

A long-debated phenomenon in the (bio)physical community is the occurrence of a dynamical transition in proteins, either globular or intrinsically disordered, which has been widely investigated by means of neutron scattering experiments [1]. As its name suggests, such a transition appears as a change of the protein dynamical properties, namely, a steep increase of their atomic mobility, due to the onset of anharmonic motions, at a temperature $T_{d}$ whose value depends on the specific system. A dynamical transition was observed at $T_{d}$ between 180 and $230 \mathrm{~K}$ for proteins such as myoglobin and lysozyme [1-3], while values up to $\simeq 240 \mathrm{~K}$ and $\simeq 260 \mathrm{~K}$ were detected for intrinsically disordered proteins [4] and in purple membranes [5], respectively. At the same $T_{d}$, the atomic motions that are responsible for protein biological function at physiological temperatures start to become active [6,7]. Much of the debate has been focused on the role played by water in the transition, with several works supporting a water-induced scenario [3,4,8-11], but also with recent findings of a dynamical transition in dry protein powders [12]. Another direction of investigation concerns the generality

\footnotetext{
*Corresponding author: emanuela.zaccarelli@cnr.it

${ }^{\dagger}$ Corresponding author: andrea.orecchini@unipg.it
}

Published by the American Physical Society under the terms of the Creative Commons Attribution 4.0 International license. Further distribution of this work must maintain attribution to the author(s) and the published article's title, journal citation, and DOI. of the phenomenon, which was found to occur in different biomacromolecules, including DNA [13], RNA [14], and lipid bilayers [15]. Along this line, recent works [16,17] reported evidence of a dynamical transition in concentrated microgel suspensions of poly $(N$-isopropylacrylamide) (PNIPAM), extending the realm of the dynamical transition to nonbiological systems. Microgels are colloidal-scale particles made by covalently cross-linked polymer networks [18], which were shown to avoid water crystallization at low temperatures even in highly hydrated samples, i.e., down to a polymer weight fraction of approximately $43 \mathrm{wt}$ \% [16]. For these systems, a clear increase of the experimental mean-squared displacement (MSD) of the polymer atoms was observed, whereas atomistic molecular dynamics simulations highlighted the pivotal role of water in the transition [17].

In the biophysical context, a dynamical transition was observed for structured proteins and also for their building blocks, namely, polypeptides [19] and even amino acids [20]. Following a similar direction, it is now legitimate to ask whether the polymeric architecture has any influence on the occurrence of such a transition by examining the case of PNIPAM linear (non-cross-linked) polymer chains. This polymer is mostly exploited for its thermoresponsive properties [21-23]: Above room temperature, PNIPAM chains undergo a reversible coil-to-globule transition with increasing temperature $T$, which makes them suitable to mimic protein folding and to investigate protein cold denaturation [24]. The similarity of PNIPAM to proteins can be traced back to its amphiphilic character and the associated complex energy landscape, endowed with multiple conformational substates of close energy [25]. Notwithstanding the wide literature on 

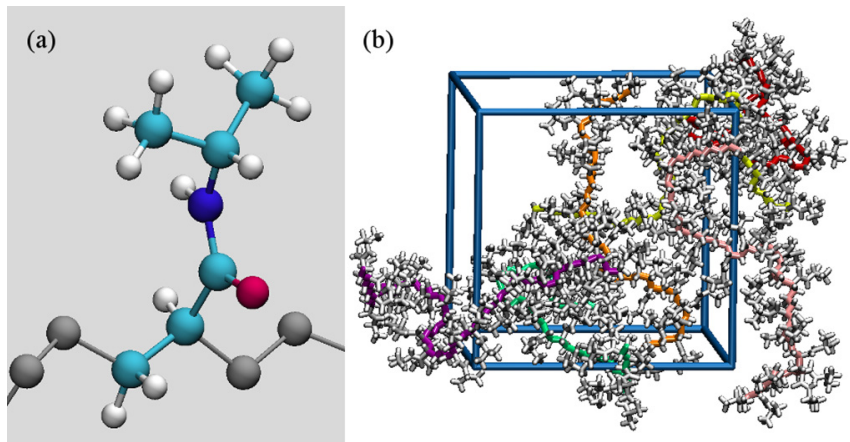

FIG. 1. (a) Chemical structure of a PNIPAM repeating unit. Carbon, oxygen, nitrogen, and hydrogen atoms are shown in light blue, red, blue, and white, respectively. Backbone carbon atoms of the polymer chain are also displayed in gray. (b) Schematic representation of the atomistic model of a suspension of PNIPAM chains in water. Backbone carbon atoms of each polymer chain are represented with a different color, while hydrogen atoms and heavy atoms in the side chain groups are displayed in gray. Water molecules are omitted for clarity.

the solution behavior of PNIPAM chains, very little is known about their properties at high concentrations and in the lowtemperature regime [26].

In this paper we provide a comprehensive investigation of the atomic dynamics of PNIPAM linear chains at low $T$, combining elastic incoherent neutron scattering (EINS) experiments at various energy resolutions and atomistic molecular dynamics (MD) simulations. Our results show the occurrence of a dynamical transition at $T_{d} \sim 225 \mathrm{~K}$, a value very similar to that observed in proteins such as myoglobin and lysozyme [1-3]. This result implies a wide generality of the phenomenon, independently of the structural details of the investigated complex macromolecular system.

\section{EXPERIMENTAL AND NUMERICAL METHODS}

\section{A. Sample preparation}

Poly( $N$-isopropylacrylamide) was purchased from Polymer Source, Inc. and used without further purification. The polymer has a molecular weight $M_{w}=189600$ and a polydispersity index of 2.88; thus each polymer chain consists of $\simeq 1669$ repeating units. Figure 1 (a) is an illustration of the chemical structure of the polymer repeating unit.

A suspension with a polymer concentration of $10 \mathrm{wt} . \%$ was prepared through three cycles of lyophilization and dispersion in $\mathrm{D}_{2} \mathrm{O}$. High-concentration samples were then obtained by filling the sample holders for neutron experiments with the dispersion at $10 \mathrm{wt} \%$ and then allowing the excess $\mathrm{D}_{2} \mathrm{O}$ to evaporate at room temperature under vacuum. The concentration was regularly monitored by accurate weighing of the samples. Once the desired concentrations of 50,60, and 95 wt. \% were reached, the holders were sealed and samples were left to homogenize for not less than four days. The sample with a polymer concentration of $95 \mathrm{wt} . \%$ is referred to as a dry sample because it corresponds to the mass fraction residue determined by thermogravimetric analysis at $430 \mathrm{~K}$.
TABLE I. The $Q$ range, energy window $\Delta E$, and probed timescale $\tau$ of the different EINS measurements. For IN16B and IN13, $\Delta E$ corresponds to the instrumental energy resolution, while for IN5 it is the $E$ window used to calculate $I(Q, 0)$. In the employed experimental configuration, the energy resolution of IN5 was $\sim 87 \mu \mathrm{eV}$ (see Appendix A).

\begin{tabular}{lcccc}
\hline \hline Parameter & IN16B & IN13 & IN5 $(1 \Delta E)$ & IN5 $(2 \Delta E)$ \\
\hline$Q$ range $\left(\AA^{-1}\right)$ & $0.2-1.9$ & $0.2-4.5$ & $0.55-2.0$ & $0.55-2.0$ \\
$\Delta E(\mu \mathrm{eV})$ & 0.75 & 8 & 97 & 191 \\
$\tau(\mathrm{ps})$ & 1800 & 150 & 15 & 7 \\
\hline \hline
\end{tabular}

\section{B. Elastic incoherent neutron scattering}

Elastic incoherent neutron scattering experiments were carried out at the neutron spectrometers IN16B, IN13, and IN5 [27] of the Institut Laue-Langevin (ILL) [28,29]. The main characteristics of the instruments are summarized in Table I, whereas the details of experiments and data reduction are thoroughly described in Appendix A.

The observable quantity in an EINS experiment is the intensity $I(Q,|E| \lesssim \Delta E) \doteq I(Q, 0)$ scattered in a narrow energy window $\Delta E$ centered about the elastic peak $E=0$, as a function of exchanged momentum $Q$. This observable was monitored as a function of $T$ with different energy windows $\Delta E$, as per Table I, allowing us to resolve atomic motions in a wide range of timescales $\tau$ from picoseconds to nanoseconds. As the neutron incoherent cross section of hydrogen exceeds by more than one order of magnitude the total (coherent plus incoherent) cross section of deuterium and other atomic species in the samples, the measured signal provides information on the dynamics of the polymer chains, while the water contribution is negligible, as reported in Table II.

\section{All-atom molecular dynamics simulations}

The in silico model of a suspension of PNIPAM linear chains in water was designed by including in a cubic box six atactic polymer segments made of 30 repeating units [30], with an extra-boundary connectivity between adjacent periodic images to mimic the effect of the higher degree of polymerization in the experimental samples, and by adding explicit water. A schematic representation of the model is

TABLE II. Characteristics of the samples for the EINS experiment on IN16B: thickness $d$ of the cells and corresponding transmission $T$ calculated for $\lambda_{i}=6.271 \AA$; calculated cross sections for the coherent, incoherent, and total scattering of each sample (PNIPAM and heavy water), $\sigma_{\text {coh }}, \sigma_{\text {inc }}$, and $\sigma_{\text {scatt }}$, respectively; and ratio between the incoherent scattering cross section of PNIPAM hydrogen atoms $\sigma_{\mathrm{H}}$ and the total scattering cross section of the sample (PNIPAM and heavy water).

\begin{tabular}{lcccccc}
\hline \hline $\begin{array}{l}\text { Sample } \\
(\text { wt. \% })\end{array}$ & $\begin{array}{c}d \\
(\mathrm{~mm})\end{array}$ & $T$ & $\begin{array}{c}\sigma_{\mathrm{coh}} \\
(\text { barn })\end{array}$ & $\begin{array}{c}\sigma_{\text {inc }} \\
(\text { barn })\end{array}$ & $\begin{array}{c}\sigma_{\text {scatt }} \\
(\text { barn })\end{array}$ & $\sigma_{\mathrm{H}} / \sigma_{\text {scatt }}$ \\
\hline 50 & 0.5 & 0.89 & 163 & 826 & 989 & 0.81 \\
60 & 0.4 & 0.89 & 128 & 817 & 945 & 0.85 \\
95 & 0.2 & 0.89 & 75 & 803 & 878 & 0.91 \\
\hline \hline
\end{tabular}



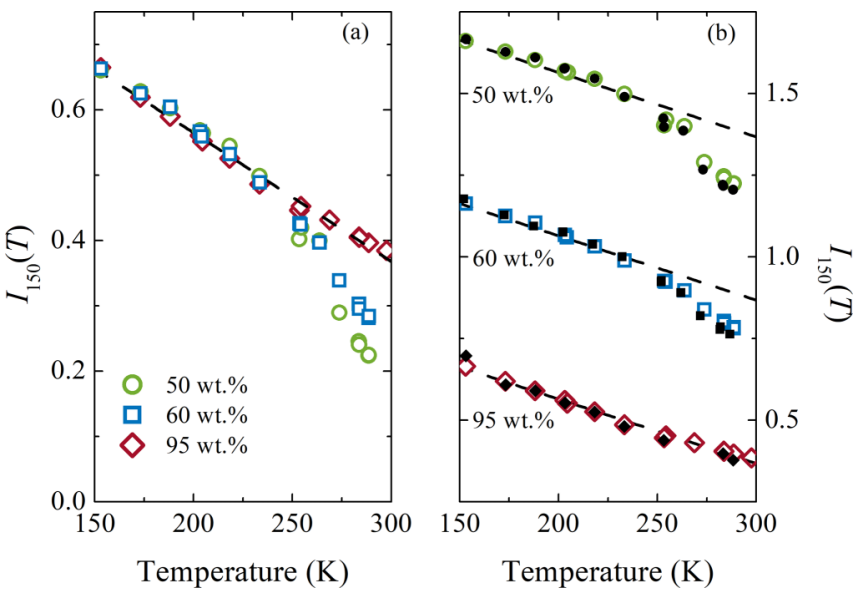

FIG. 2. Plot of IN13 integrated elastic intensity $I_{150}(T)$ as a function of temperature for (a) chains as a function of PNIPAM concentration and (b) PNIPAM chains (open symbols) compared to PNIPAM microgels (closed symbols). The dashed black lines are linear fits to the data of the dry polymer chain. Data are normalized to 1 for $T \rightarrow 0$. In (b), data at different concentrations are shifted by 0.5 for clarity. Error bars are within symbol size.

shown in Fig. 1(b). All-atom MD simulations were performed on linear polymer chains suspensions with a PNIPAM mass fraction of $60 \%$ using the GROMACS 5.1.4 software [31,32]. PNIPAM and water were described using the optimized potentials for liquid simulation all-atom force field [33] with the implementation by Siu et al. [34] and the TIP4P/Ice model [35], respectively. Numerical simulations were carried out in a wide range of temperatures, by cooling the system from 293 to $193 \mathrm{~K}$. This allows a direct comparison with experiments. At each temperature the system was first equilibrated in a pressure bath at 1 bar, maintained by the Parrinello-Rahman barostat with a time constant of $2 \mathrm{ps}$, up to a constant density value, i.e., drift less than $2 \times 10^{-3} \mathrm{~g} \mathrm{~cm}^{-3}$ over $20 \mathrm{~ns}$. Simulation data were then collected for $330 \mathrm{~ns}$ in the NVT ensemble, with a sampling of 0.2 frame/ps.

The leapfrog integration algorithm was employed with a time step of 2 fs. The length of bonds involving hydrogen atoms was kept fixed with the LINCS algorithm. Cubic periodic boundary conditions and minimum image convention were applied. The temperature was controlled with the velocity rescaling thermostat [36] with a time constant of 0.1 ps. Electrostatic interactions were treated with the smooth particle-mesh Ewald method with a cutoff of nonbonded interactions of $1 \mathrm{~nm}$. A total trajectory interval of about $0.5 \mu \mathrm{s}$ was calculated for each temperature. The last $300 \mathrm{~ns}$ of the trajectory were considered for analysis. The software VMD [37] was employed for graphical visualization.

\section{RESULTS}

We start by reporting the integrated elastic intensity $I_{\tau}(T)$, i.e., the integral over the whole measured $Q$-range of $I(Q, 0)$ at a given $\tau$, as a function of temperature. Figure 2(a) shows $I_{150}(T)$ measured at IN13 for different polymer weight fractions. While the dry system displays a linear decrease of $I_{150}(T)$ with increasing $T$, the intensity of the two hydrated

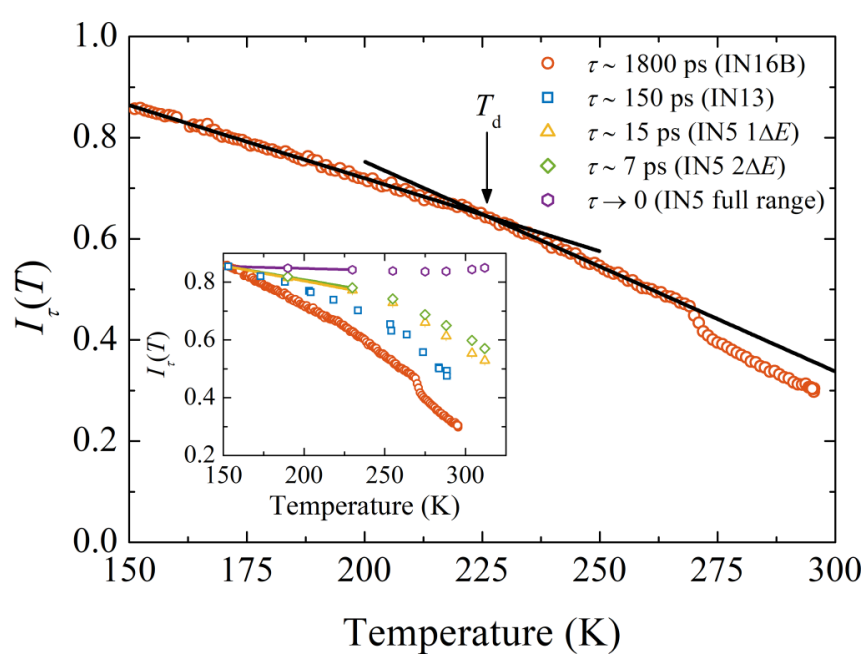

FIG. 3. Plot of IN16B integrated elastic intensity of 60 wt. \% linear chains. The dynamical transition temperature $T_{d}=226 \pm 1 \mathrm{~K}$ is determined as the crossing point of two linear behaviors (black lines). The inset shows $I_{\tau}(T)$ obtained by integrating $I(Q, 0)$ measured at different $\tau$ in the common $Q$ range from 0.6 to $1.8 \AA^{-1}$. The IN13 data are normalized to IN16B data at $150 \mathrm{~K}$; for IN5 data, normalization is obtained through a linear extrapolation of the lowest- $T$ measurements (solid lines).

samples undergoes a clear drop at a temperature that can be located roughly within 225 and $250 \mathrm{~K}$. The departure from the linear trend marks the onset of anharmonic motions ascribed to the occurrence of the dynamical transition [1]. Within the available $T$-resolution, $T_{d}$ does not show a dependence on sample concentration, while the extent of the drop in $I_{150}(T)$ does. Hence, for the dry sample the transition disappears, in agreement with studies suggesting a driving role of water in the transition $[4,8,38]$. These findings are in quantitative agreement with IN13 measurements on PNIPAM microgel networks [16], reported for comparison in Fig. 2(b). The nearly identical $T$-behavior for $I_{150}(T)$ observed for polymer chains and microgels at corresponding concentrations suggests that the mesoscopic topological features of the macromolecule, such as molecular weight and polydispersity, have negligible effects on the phenomenon. Therefore, we conclude that there is no difference between cross-linked and linear chains, highlighting the fact that the dynamical transition does not depend on the details of the internal macromolecular architecture, similarly to what was observed for biological systems.

Since there is no strong dependence of $T_{d}$ on wt. $\%$, from now on we focus on the $60 \mathrm{wt} \%$ sample only. Figure 3 shows the integrated elastic intensity $I_{1800}(T)$ measured at IN16B. Its higher flux allows us to obtain a finer temperature sampling than IN13, thus revealing three different regimes: (i) a linear decrease above $150 \mathrm{~K}$, which in proteins is known to account for both harmonic movements and methyl group rotation [39,40]; (ii) a second, steeper linear decrease above $T_{d} \sim 225 \mathrm{~K}$, which marks the dynamical transition; and (iii) a third, sudden drop around $270 \mathrm{~K}$. The latter, which is not clearly separated from (ii) on the less resolved $T$-scan performed on IN13, might be related to ice melting. To shed light on this issue, we performed complementary differential 
scanning calorimetry (DSC) measurements (see Appendix B), which confirmed the absence of macroscopic crystallization upon cooling $[16,26]$ and the onset of a process of cold crystallization upon heating, involving only about $2.5 \%$ of the water molecules. As shown in Appendix B 2, this overall behavior is consistent with both cooling and heating cycles of the IN16B measurements.

The finer temperature sampling allows us also to determine with greater accuracy that the dynamical transition in linear chains takes place at about $225 \mathrm{~K}$, well below the value of $250 \mathrm{~K}$ that was initially suggested by IN13 for microgel systems $[16,17]$. Given that microgels and linear chains display identical behavior on IN13, we expect the true $T_{d}$ of microgels to be close to $225 \mathrm{~K}$ too.

The inset of Fig. 3 shows a comparison of all intensities $I_{\tau}(T)$ obtained by integrating $I(Q, 0)$ measured at the various $\tau$ on a common $Q$-range. Qualitatively, the data show a less and less pronounced intensity decrease as $\tau$ gets shorter, ending up in a flattening of the curve when the static approximation $\tau \rightarrow 0$ is approached. All curves at finite $\tau$ exhibit a change of slope around $\sim 225 \mathrm{~K}$. Hence, the present data do not suggest a clear dependence of $T_{d}$ on the experimental resolution, at variance with previous reports for hydrated protein powders [41]. This discrepancy might be due either to a true difference between PNIPAM and proteins or possibly to the sparse $T$ sampling of our IN13 and IN5 data.

For a quantitative analysis of the $T$-evolution of the polymer dynamics, we use the widely employed double-well model for incoherent elastic scattering [1,42], which assumes that the sample hydrogen atoms can jump between two distinct sites of different free energy and separated by a distance $d$. Hence, $I(Q, 0)$ can be written as

$$
I(Q, 0)=I_{0} e^{-b Q^{2}}\left[1-2 p_{1} p_{2}\left(1-\frac{\sin (Q d)}{Q d}\right)\right],
$$

where $I_{0}$ is an intensity prefactor, $b=\left\langle\Delta u^{2}\right\rangle_{\text {vib }}$ is the harmonic vibrational MSD of an atom moving within a single well, and $p_{1}$ and $p_{2}$ are the probabilities of finding the atom in the first or second well, respectively. For a robust determination of the physical parameters of interest, we set up a global analysis procedure that simultaneously fits the data acquired at all the measured timescales, over the three different $Q$-ranges and over all temperatures (see Appendix C). Specifically, we assume that (i) the harmonic behavior of our samples is faster than any of the experimental timescales $\tau$ in Table I, thus being equally resolved by the three employed spectrometers, and (ii) upon increasing $\tau$, hydrogen atoms can explore a wider spatial region, and hence $d$ increases. Consequently, we constrain $\left\langle\Delta u^{2}\right\rangle_{\text {vib }}$ to follow a linear behavior in $T$ with $\tau$-independent parameters, whereas $I_{0}$ and $p_{1} p_{2}$ are free to vary with both $\tau$ and $T$. In this way, we find that $d$ is roughly constant in $T$ but grows with $\tau$ as $d(\tau)=\phi \tau^{\xi}$, in agreement with previous findings for proteins [41]. We thus constrain $d$ to follow such a power-law behavior with $\phi$ and $\xi$ as additional fit parameters. From this model we can finally estimate the total MSD [42] of PNIPAM hydrogen atoms for each time resolution, as

$$
\mathrm{MSD}=6\left\langle\Delta u^{2}\right\rangle_{\mathrm{vib}}+2 p_{1} p_{2} d^{2} .
$$

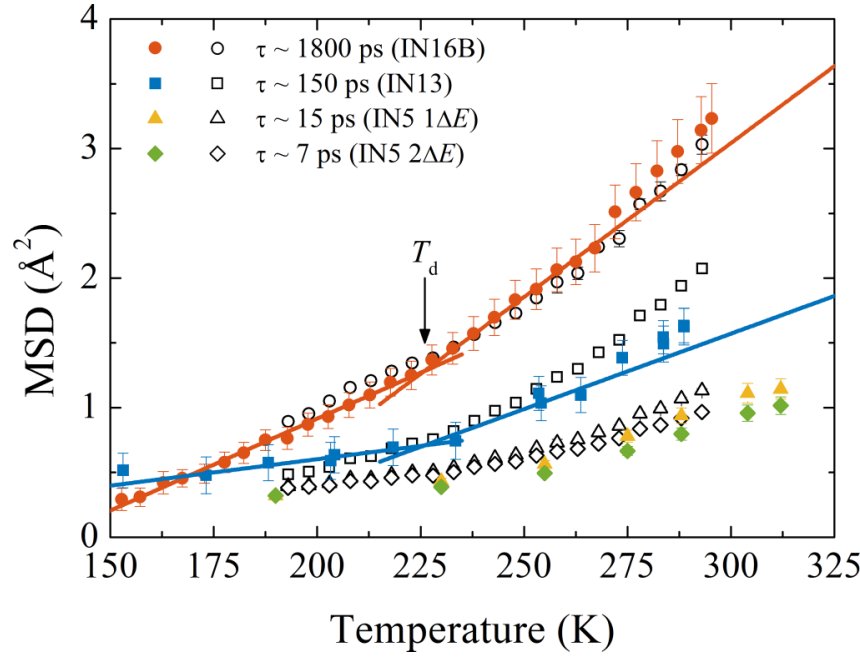

FIG. 4. Comparison between experimental MSDs (closed symbols), obtained by fitting $I(Q, 0)$ as described in the text, and numerical MSDs (open symbols) at the same timescales. The transition temperature obtained from the integrated elastic intensity is marked with a black arrow. Red and blue lines are guides to the eye to highlight the transition in IN16B and IN13 data, respectively. Error bars amount to one standard deviation and when not visible are within the symbol size. The high value of the first IN13 point at $150 \mathrm{~K}$ may be due to fluctuations in the data normalization reference, but it does not affect the change of slope of the data at higher temperatures.

The resulting MSDs are reported in Fig. 4 as a function of temperature at the various experimental timescales. As expected, at a given temperature MSDs are larger for longer observation timescales $\tau$. Considering IN16B and IN13 data, we easily recognize the same three regimes also observed for the integrated elastic intensity in Fig. 3. Our results do not show a clear evolution of $T_{d}$ with $\tau$. In proteins, the existence of this dependence is debated and results range from a strong variation of $T_{d}$ [2] to a substantially $\tau$-independent transition [41]. In our case, we find that $T_{d} \sim 225 \mathrm{~K}$ seems compatible with all data sets, although the sparse $T$-sampling of IN13 and IN5 does not allow us to unambiguously determine a possible trend.

It is now instructive to compare the experimental results with MD simulations. The MSDs of PNIPAM hydrogen atoms directly evaluated from MD simulations at each time resolution as a function of $T$ are also reported in Fig. 4. Remarkably, the numerical data quantitatively agree with the experimental estimates at all temperatures and for all measured time resolutions, without any arbitrary scaling factor. This confirms previous results obtained from IN13 measurements of microgels [16], crucially extending their validity by more than two decades in time. In addition, the direct comparison of experiments and simulations strongly validates the global fit procedure that we have adopted to extract experimental MSDs from the measured elastic intensities. Indeed, we stress that a free fit would not be able to provide the same level of consistency both between data measured at different $E$-resolution and $Q$-range and between experimental and numerical data. Furthermore, these findings corroborate the use of the TIP4P/Ice model, which fully captures the $T$ 

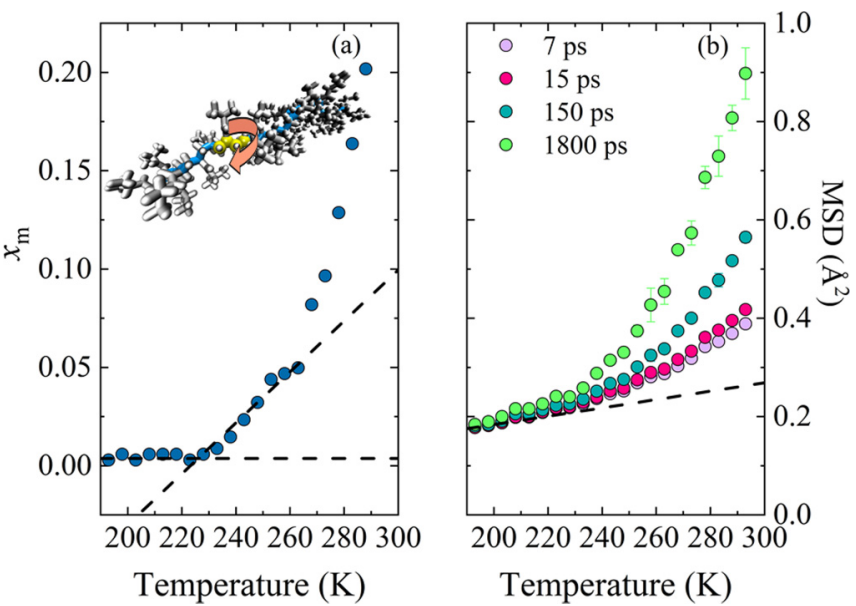

FIG. 5. Plot of MD results for (a) fraction of mobile backbone dihedral angles $x_{m}$ and (b) MSD of hydrogen backbone atoms for different time resolutions, both as a function of $T$. A snapshot of a polymer chain is also shown in (a), with backbone atoms in blue and a dihedral angle highlighted in yellow. In both panels, dashed lines are guides to the eye to indicate the occurrence of the dynamical transition at $T_{d} \sim 225 \mathrm{~K}$. When not visible, error bars are within symbol size.

dependence of the dynamical behavior of polymer atoms, and thus emerges as the optimal water model to simulate PNIPAM dynamics in supercooled water.

The numerical MSD data substantiate the behavior of the integrated elastic intensity and also point to the occurrence of a dynamical transition for linear PNIPAM chains around $T_{d} \sim 225 \mathrm{~K}$. While simulations have been performed with a temperature mesh of $5 \mathrm{~K}$, due to the long simulation time required for each state point, this sampling is sufficient to locate $T_{d}$ in good agreement with experiments. We remark that a different value of $T_{d}$, roughly around $250 \mathrm{~K}$, was previously reported for microgels $[16,17]$, but this larger value can be entirely ascribed to the broader $T$-sampling used in such earlier studies.

The simulations further allow us to identify the specific motions underlying the onset of the transition, which should be connected, as in proteins [43], to the activation of local segmental motions. This is investigated by monitoring the fraction of mobile backbone dihedral angles $x_{m}$ as a function of $T$, which is shown in Fig. 5(a). We find that, compatibly with statistical uncertainty, $x_{m}$ becomes larger than zero for $T \gtrsim 230 \mathrm{~K}$, thus confirming the molecular origin of the transition with the activation of bond flips between rotational isomeric states of the chain backbone. We stress that this analysis has been performed on the whole equilibrated run, so its results can be considered to be independent of time resolution. Finally, it is important to note that while methyl hydrogen atoms are active at all investigated temperatures (see Appendix D), backbone hydrogen atoms are inactive below $T_{d}$, as shown by their MSDs reported as a function of $T$ in Fig. 5(b) for different values of $\tau$. Clearly, deviations from a low- $T$ linear regime again occur only for temperatures higher than $225 \mathrm{~K}$. These results confirm that, also in the simulation data, the value of $T_{d}$ is independent of the probed time window.

\section{CONCLUSION}

In this work we reported evidence of a low-temperature dynamical transition in linear PNIPAM polymer chains by means of EINS measurements at resolutions covering more than two decades in time. A global fit, based on the doublewell model, was simultaneously applied to all measured data as a function of $T, Q$, and $\Delta E$, allowing us to extract the MSDs of PNIPAM hydrogen atoms. These were found to be in quantitative agreement with those calculated in atomistic MD simulations, in the whole resolution interval and without any scaling factor. Our results clearly indicate that the dynamical transition takes place, in both linear chains and cross-linked microgels, at $T_{d} \sim 225 \mathrm{~K}$, like in globular proteins. The value of $T_{d}$ seems to be independent of the experimental resolution, although further studies are needed to fully clarify this point. We thus confirm the occurrence of a dynamical transition in hydrated powders of nonbiological systems [16,44], further indicating that this phenomenon is not dictated by the internal polymer architecture. It rather emerges as a general process, in those macromolecular systems that are characterized by dynamical complexity, due to multiple stable configurations of similar energy, and capable of efficiently confining water, coupling with it by hydrogen bonding [17], and thus avoiding ice crystallization. These findings further challenge the present understanding of the relationship between the dynamical behavior and biological function of a biomacromolecule, and thus call for deeper investigation of the molecular mechanisms underlying the dynamical transition.

\section{ACKNOWLEDGMENTS}

We thank Roberta Angelini and Silvia Franco for their help in performing the DSC characterization of our samples. We acknowledge ILL for beamtime and CINECA-ISCRA for computer time. L.T., E.C., and E.Z. acknowledge support from European Research Council (Grant No. 681597 MIMIC); L.T., E.B., E.C., and E.Z. from MIUR (FARE Project No. R16XLE2X3L, SOFTART); and E.B., E.C., and E.Z. from Regione Lazio, through L.R. 13/08, Progetto Gruppo di Ricerca GELARTE Project No. prot.85-201715290. The open access fee was covered by FILL2030, a European Union project within the European Commission's Horizon 2020 Research and Innovation programme under Grant Agreement No. 731096.

L.T. and M.Z. contributed equally to this work.

\section{APPENDIX A: EINS EXPERIMENTS AND DATA TREATMENT}

Elastic incoherent neutron scattering experiments were carried out loading the samples inside flat aluminium cells $(3.0 \times$ $4.0 \mathrm{~cm}^{2}$ ) sealed with an In O-ring. The thickness of each cell was selected to achieve a transmission of about $90 \%$ at an incoming wavelength $\lambda_{i}=6.271 \AA$ (see Table II). The weight of each sample was checked before and after each measurement without observing any appreciable variation. Considering the total, coherent, and incoherent scattering cross sections [45], the signal measured for each sample is largely dominated by the incoherent contribution of PNIPAM hydrogen atoms, ranging from $81 \%$ to $91 \%$ (see Table II). 

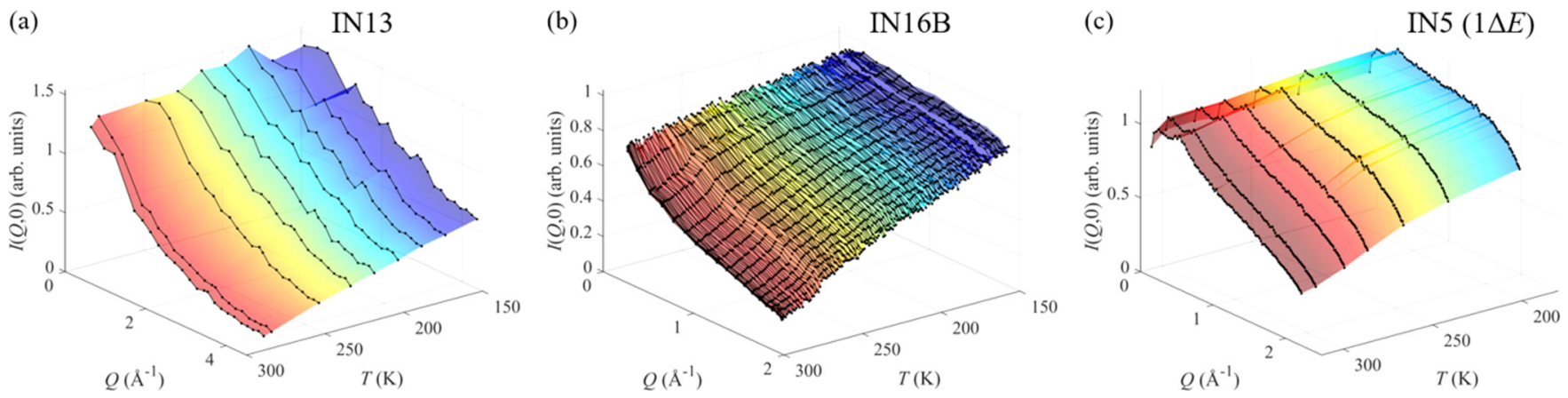

FIG. 6. Temperature evolution of the elastic incoherent intensity $I(Q, 0)$ for the $60 \mathrm{wt} . \%$ sample measured using different instruments: (a) IN16B upon heating, (b) IN13 upon cooling, and (c) IN5 upon heating and after integration over $1 \Delta E$. The colormap from blue to red indicates the increasing temperature.

\section{IN13 data}

IN13 is a high-resolution backscattering spectrometer using thermal neutrons. In the elastic configuration, IN13 operates with an incident wavelength $\lambda_{i}=2.23 \AA$ and covers an interval of exchanged momentum $Q$ from about 0.2 to $4.5 \AA^{-1}$, with an energy resolution of $8 \mu \mathrm{eV}$, obtained as the full width at half maximum (FWHM) of a Gaussian fit to the elastic peak of a standard vanadium sample. Measurements were carried out on the 50,60, and 95 wt. \% PNIPAM linear chain samples. The $I(Q, 0)$ was acquired at selected fixed temperatures, cooling the sample from about $290 \mathrm{~K}$ down to $150 \mathrm{~K}$ and then heating back to room temperature. The acquisition time for each temperature ranged from $30 \mathrm{~min}$ to $2 \mathrm{~h}$.

Data were corrected for incident flux, cell scattering, and self-shielding. The $I(Q, 0)$ of each sample was normalized to a vanadium standard to account for detector efficiency fluctuations. Given the atomic composition, density, and geometry of our samples, the ratio of multiple-to-single total scattering (i.e., integrated over the whole $(Q, E)$ space) can be evaluated to amount to about $16 \%$. In the restricted elastic window and reduced $Q$ range of interest, multiple scattering reduces to a smaller fraction and, in mainly incoherent samples like ours, it is rather structureless as a function of $Q$. Therefore, it cannot affect results such as the temperature behavior of the data and the value of the dynamical transition temperature $T_{d}$. Consequently, multiple scattering corrections were neglected. Figure 6(b) shows the so-obtained $I(Q, 0)$ for the 60 wt. \% PNIPAM linear chain sample.

\section{IN16B data}

Measurements at the high-flux backscattering spectrometer IN16B were performed in the $\mathrm{Si}(111)$ configuration, which produces an elastic $E$ resolution of $0.75 \mu \mathrm{eV}$ (FWHM) using neutrons with an incident wavelength $\lambda_{i}=6.271 \AA$ A. Data were acquired over a $Q$-range from about 0.2 to $1.9 \AA^{-1}$. The $I(Q, 0)$ was measured on the $60 \mathrm{wt} . \%$ sample during a heating ramp with a controlled heating rate of $0.3 \mathrm{~K} / \mathrm{min}$. The acquisition time was $30 \mathrm{~s}$ per temperature.

Data were corrected for incident flux, cell scattering, and self-shielding. Each $I(Q, 0)$ was normalized to a lowtemperature measurement of the sample. As explained above, multiple scattering corrections were neglected. Figure 6(a) shows the so-obtained $T$ evolution of the $I(Q, 0)$ for the 60 wt. \% PNIPAM linear chains sample.

\section{IN5 data}

The time-of-flight (TOF) spectrometer IN5 allows measurements of the full dynamic structure factor $S(Q, E)$ over a broad $(Q, E)$-range. The instrument was configured to select neutrons of incident wavelength $\lambda_{i}=5 \AA$, with a chopper speed of $12000 \mathrm{rpm}$. This provided an energy resolution of $87 \mu \mathrm{eV}$ (FWHM). Measurements were carried out on the 60 wt. \% sample. Data were acquired at selected fixed temperatures, heating the sample from about 190 up to $312 \mathrm{~K}$. The acquisition time for each temperature was $1 \mathrm{~h}$.

The two-dimensional detector of IN5 collected the scattered neutron intensity as a function of detector pixel position $(x, y)$, which defined the scattering angle $2 \theta$, and neutron TOF. Given the isotropic nature of the sample, different $(x, y)$ pixels corresponding to the same $2 \theta$ were rebinned together into suitably spaced Debye-Scherrer cones. Data were corrected for incident flux, cell scattering, and self-shielding, then normalized to a vanadium standard, and finally converted from TOF to exchanged energy, thus obtaining $I(2 \theta, E)$ spectra. Once again, multiple scattering processes were neglected.

To extract the elastic intensity of interest, the $I(2 \theta, E)$ spectra were integrated over two different symmetrical regions around the elastic peak, respectively, with extensions $1 \Delta E=97 \mu \mathrm{eV}$ and $2 \Delta E=191 \mu \mathrm{eV}$. Finally the scattering angle $2 \theta$ was converted into $Q$, leading to the elastic intensities $I(Q, 0)$ shown in Fig. 6(c). Due to the intersection geometry of the Debye-Scherrer cones with the detector surface, nonphysical intensity drops appear at the lowest and highest $Q$-values, namely, for $Q$ smaller than $0.55 \AA^{-1}$ and larger than $2.0 \AA^{-1}$ (see Fig. 11). The corresponding data points were discarded during the data fitting procedure.

\section{APPENDIX B: COLD CRYSTALLIZATION AND MELTING}

\section{DSC analysis}

Thermal analyses (Fig. 7) on PNIPAM chains at 60 wt. \% were recorded with a differential scanning calorimeter DSC 8000 Perkin Elmer, equipped with Intracooler II as the cooling system. 

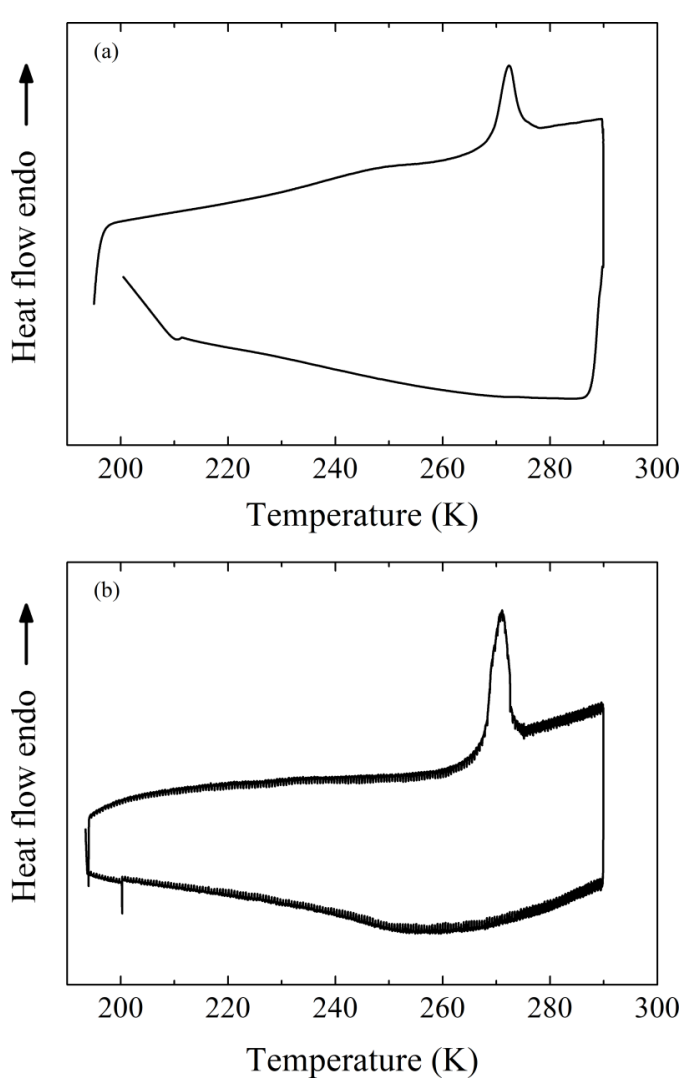

FIG. 7. Plot of DSC thermograms of PNIPAM dispersions at $60 \mathrm{wt}$. $\%$, measured at (a) $10 \mathrm{~K} / \mathrm{min}$ and (b) $0.3 \mathrm{~K} / \mathrm{min}$.

DSC analyses were done on about $10-15 \mathrm{mg}$ of PNIPAM dispersion at a concentration of 60 wt. $\%$ in $\mathrm{D}_{2} \mathrm{O}$. Measurements were carried out under nitrogen atmosphere ( $20 \mathrm{ml} / \mathrm{min}$ ) in a sealed pan of aluminium to prevent changes in concentration during the heating/cooling steps. Samples were prepared starting from the material used in EINS experiments. PNIPAM linear chains were dried up and then dispersed again in $\mathrm{D}_{2} \mathrm{O}$ to obtain a concentration of $10 \mathrm{wt}$. \%. The target concentration was reached by evaporating the excess $\mathrm{D}_{2} \mathrm{O}$ and then pans were sealed and analyzed.

The measurement shown in Fig. 7(a) was carried out by cooling the system from 298 to $193 \mathrm{~K}$ at $40 \mathrm{~K} / \mathrm{min}$, then heating it to $290 \mathrm{~K}$, and finally cooling it again to $193 \mathrm{~K}$ with a scanning rate of $10 \mathrm{~K} / \mathrm{min}$. The measurement reported in Fig. 7(b) was carried out by cooling the system from 298 to $193 \mathrm{~K}$ with a scanning rate of $40 \mathrm{~K} / \mathrm{min}$, then heating back to $290 \mathrm{~K}$, and finally cooling again to $193 \mathrm{~K}$ with a scanning rate of $0.3 \mathrm{~K} / \mathrm{min}$. The first cooling steps at $40 \mathrm{~K} / \mathrm{min}$ are not reported here. The second protocol was followed to simulate the thermal history of the samples during the EINS experiments.

Overall, the two thermograms obtained at different scanning rates are very similar, with the same $T_{\text {peak }}$ at $271 \mathrm{~K}$. In both cases, no crystallization peaks were detected under cooling, even at the lower scanning rate. However, during the heating step, a process of cold crystallization (exothermic peak) followed by fusion (endothermic peak) was observed, thus indicating the presence of a small amount of crystallized $\mathrm{D}_{2} \mathrm{O}$, following glass melting upon reheating. The values of $T_{\text {onset }}, T_{\text {peak }}$, and the melting enthalpy $\Delta H_{m}$, extracted from
TABLE III. Onset $T_{\text {onset }}$ and melting temperature $T_{\text {peak }}$, melting enthalpy $\Delta H_{m}$ of $\mathrm{D}_{2} \mathrm{O}$, and degree of crystallinity $X_{c}$ in PNIPAM $60 \mathrm{wt} . \%$ for two different scanning rates.

\begin{tabular}{lcccc}
\hline \hline $\begin{array}{l}\text { Scan rate } \\
(\mathrm{K} / \mathrm{min})\end{array}$ & $\begin{array}{c}T_{\text {onset }} \\
(\mathrm{K})\end{array}$ & $\begin{array}{c}T_{\text {peak }} \\
(\mathrm{K})\end{array}$ & $\begin{array}{c}\Delta H_{m} \\
(\mathrm{~J} / \mathrm{g})\end{array}$ & $\begin{array}{c}X_{c} \\
(\%)\end{array}$ \\
\hline 10 & 268.7 & 272.3 & 7.54 & 0.9 \\
0.3 & 270.7 & 271.1 & 20.89 & 2.5 \\
\hline \hline
\end{tabular}

the onset, the maximum of the peak, and the area of the peak, respectively, are reported in Table III.

The peak at $\sim 270 \mathrm{~K}$ confirms the hypothesis that the sudden drop observed in the integrated elastic intensity $I_{1800}(T)$ measured by IN16B is related to ice melting. The degree of crystallinity $X_{c}$ in the dispersions can be calculated, using the enthalpy obtained from the peak area, with the equation

$$
X_{c}(\%)=\frac{\Delta H_{m}}{\Delta H_{m}^{0}} \cdot \mathrm{D}_{2} \mathrm{O}(\text { wt. \% })
$$

where $\Delta H_{m}^{0}$ is the standard fusion enthalpy of deuterium oxide at $276.7 \mathrm{~K}$, which is $340.7 \mathrm{~J} / \mathrm{g}$. The degree of crystallinity
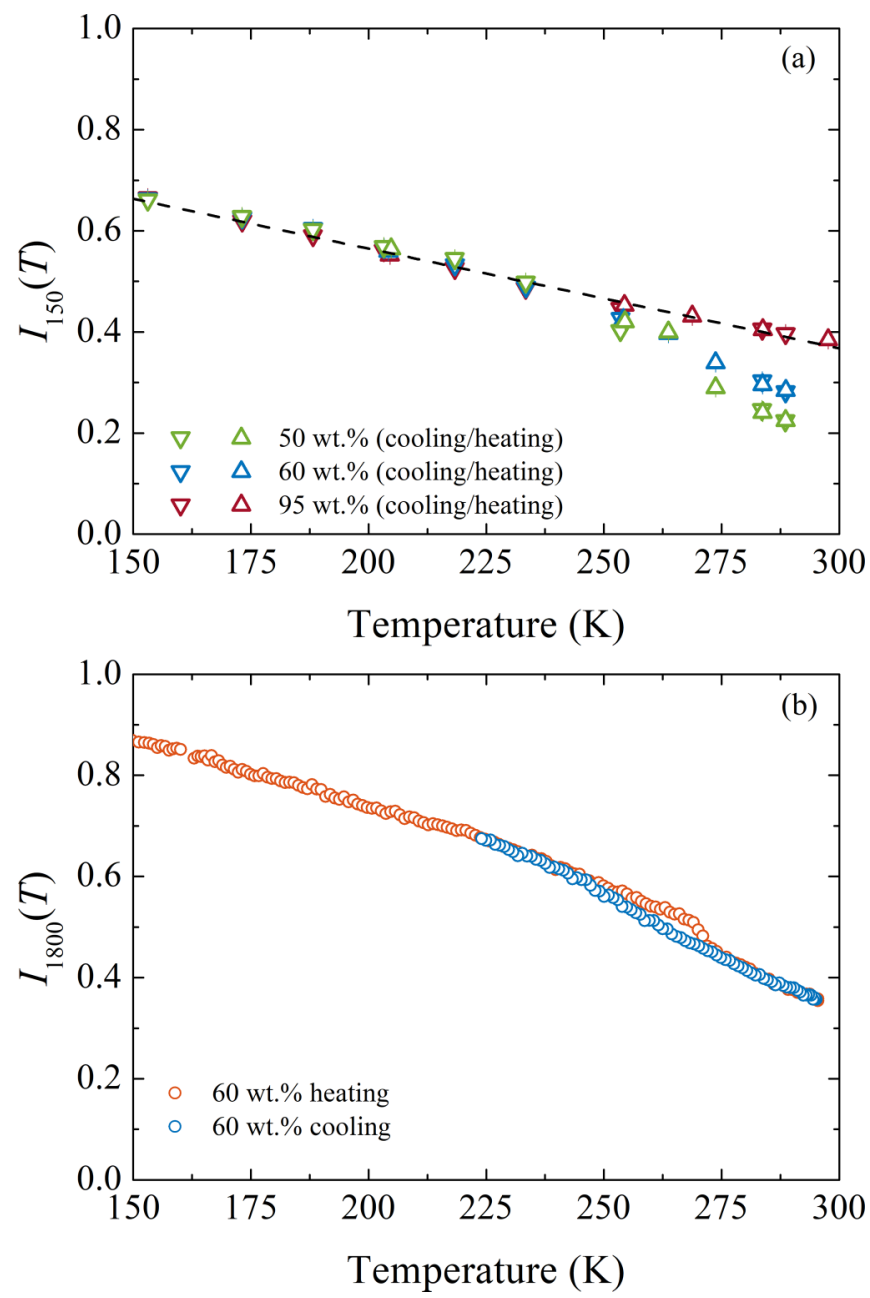

FIG. 8. Comparison between the $Q$-integrated intensities acquired during cooling and heating cycles on (a) IN13 and (b) IN16B. 


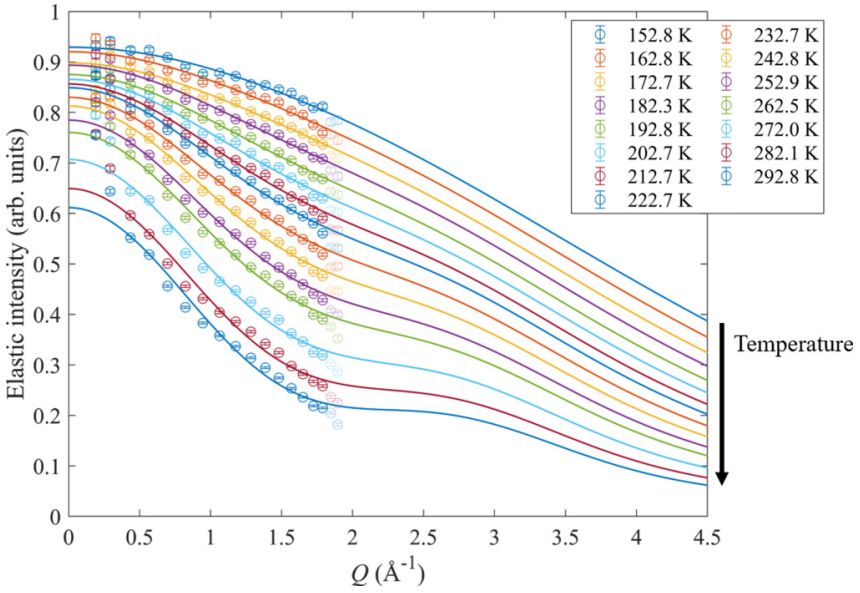

FIG. 9. Typical fits of the $I(Q, 0)$ measured on IN16B in PNIPAM linear chains at 60 wt. \%. For the sake of clarity, only selected temperature are reported. Shaded data were not used in the fit procedure. Colors are defined in the legend and the black arrow indicates the direction of the increasing temperature.

is reported in Table III and shows a crystallization of only a very small fraction of heavy water. It is worth noting that $X_{c}$ measured in Fig. 7(b) is slightly higher than that of Fig. 7(a), probably due to the lower scanning rate.

\section{Comparison between cooling and heating scans in EINS experiments}

The IN13 and IN16B data were measured both upon cooling and upon heating. As described in Appendixes A 1 and A 2 , on IN13 the $I(Q, 0)$ was acquired in static mode at fixed temperature steps for $1 \mathrm{~h}$ each, whereas on IN16B the $I(Q, 0)$ was saved every $30 \mathrm{~s}$ along a dynamic temperature scan with constant heating or cooling rate. The resulting integrated intensities are compared in Fig. 8. While on IN13 [Fig. 8(a)] there is no difference between cooling and heating cycles, a small but clear hysteresis appears in the IN16B data [Fig. 8(b)].

The absence of hysteresis on IN13 is due to both the much longer acquisition time and the static temperature mode adopted for the measurements. Both experimental choices are imposed by the lower neutron flux of IN13. As a consequence, the sample is allowed for long equilibration times at each measured temperature and is always in thermodynamic equilibrium, which results in perfectly overlapping heating and cooling data points. This is not the case for the IN16B data, where the temperature ramp mode with very fast acquisition times reveals the effects of possible temperature gradients in the sample and the details of its thermodynamic evolution. Indeed, a sudden intensity drop is observed in the heating cycle around $271 \mathrm{~K}$, which is not present along the cooling cycle. The heating and cooling curves merge again around $240 \mathrm{~K}$.

The hysteresis observed for IN16B data is actually in very good agreement with the calorimetric data reported in Fig. 7. Indeed, it is clear that the intensity drop at $271 \mathrm{~K}$, witnessing a sudden increase of atomic mobility, is consistent with the sharp peak in the heating DSC ramp due to the fusion of the

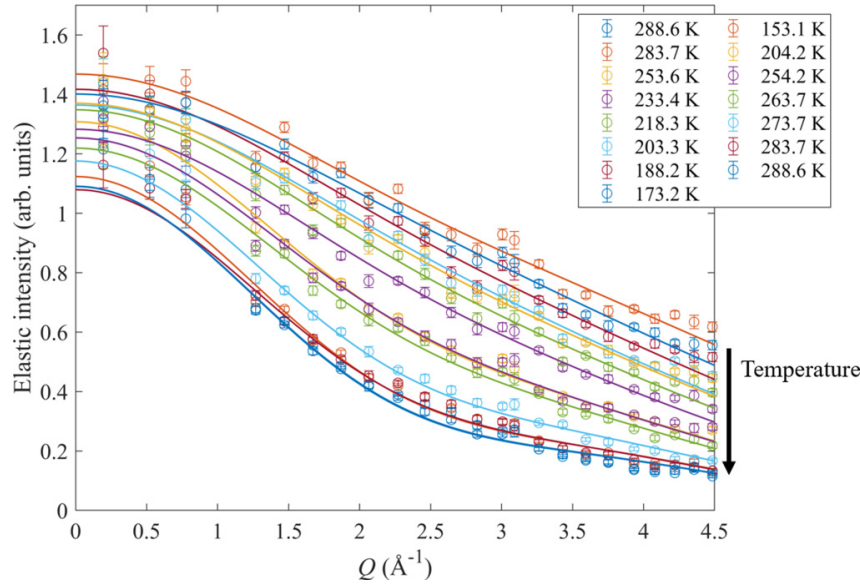

FIG. 10. Typical fits of the $I(Q, 0)$ measured on IN13 in PNIPAM linear chains at $60 \mathrm{wt} . \%$. Colors are defined in the legend and the black arrow indicates the direction of the increasing temperature.

small amount of cold-crystallized water reported in Table III. Instead, the smooth behavior of EINS data upon cooling is reflected by the cooling DSC ramp where no crystallization peaks are present.

\section{APPENDIX C: EINS DATA ANALYSIS AND FIT PROCEDURE}

As thoroughly described in Sec. III, EINS data were fitted using the double-well model of Eq. (1) $[1,42]$.

The data acquired at the four different energy resolutions, over the three different $Q$-ranges, and over all temperatures were fitted simultaneously within a global fit procedure. To do so, we assumed that (i) $I_{0}$ depends on both $\tau$ and $T$, so it is a local parameter for each $I(Q, 0)$; (ii) $b$ does not depend on $\tau$, while following a linear dependence on $T$, and then $b(T)=q+m T$ with $q$ and $m$ global parameters common to all the data sets; (iii) $p_{1}$ and $p_{2}$ depend both on $\tau$ and $T$, so they are local parameters for each $I(Q, 0)$; (iv) $d$ does not

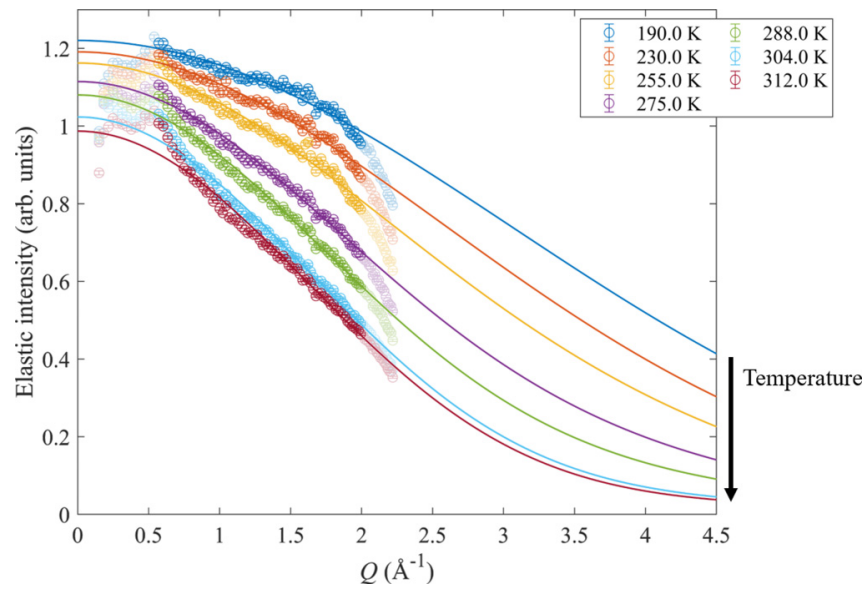

FIG. 11. Typical fits of the $I(Q, 0)$ measured on IN5 in PNIPAM linear chains at 60 wt. $\%$. Shaded data were not used in the fit procedure. Colors are defined in the legend and the black arrow indicates the direction of the increasing temperature. 
TABLE IV. Torsional dynamics of the polymer dihedral angles. Here $T$ is the temperature and $x_{m}$ is the percentage of mobile dihedrals of the backbone and the methyl groups. The analysis is carried out over the last $300 \mathrm{~ns}$.

\begin{tabular}{lcccccc}
\hline \hline & \multicolumn{2}{c}{$\boldsymbol{x}_{\boldsymbol{m}}$} & & \multicolumn{2}{c}{$\boldsymbol{x}_{\boldsymbol{m}}$} \\
\cline { 2 - 3 }$T(\mathrm{~K})$ & Backbone & Methyl & $T(\mathrm{~K})$ & Backbone & Methyl \\
\hline 193 & 0.29 & 100 & 243 & 2.3 & 100 \\
198 & 0.58 & 100 & 248 & 3.2 & 100 \\
203 & 0.29 & 100 & 253 & 4.4 & 100 \\
208 & 0.58 & 100 & 258 & 4.7 & 100 \\
213 & 0.58 & 100 & 263 & 5.0 & 100 \\
218 & 0.58 & 100 & 268 & 8.2 & 100 \\
223 & 0.29 & 100 & 273 & 9.6 & 100 \\
228 & 0.58 & 100 & 278 & 13 & 100 \\
233 & 0.88 & 100 & 283 & 16 & 100 \\
238 & 1.5 & 100 & 288 & 20 & 100 \\
\hline \hline
\end{tabular}

depend on $T$; and (v) the dependence of $d$ on $\tau$ can be written as a power law of the form $d=\phi \tau^{\xi}$ [41], where $\phi$ is related to the diffusion coefficient, while the exponent $\xi<0.5$ takes into account a possible subdiffusive behavior often observed in polymeric systems [46]. Results for the $60 \mathrm{wt} \% \%$ sample are shown in Figs. 9-11. Within this approach, the model describes very well our data, providing also good agreement with the simulations (see Fig. 4).

To improve the signal to noise ratio, the $I(Q, 0)$ acquired on IN16B were binned over $T$ channels of $5 \mathrm{~K}$. The temperature of the final data is the average temperature of the $I(Q, 0)$ inside each $T$ channel.

\section{APPENDIX D: PNIPAM INTERNAL DYNAMICS FROM MOLECULAR DYNAMICS SIMULATIONS}

We investigate the onset of anharmonic motions in the polymer chains by monitoring the conformation and torsional dynamics of dihedral angles. In the analysis of the torsional dynamics of methyl groups in PNIPAM side chains, we defined the dihedral of the methyl group as the angle formed by the atoms $\mathrm{N}-\mathrm{C}_{\text {isopropyl }}-\mathrm{C}_{\text {methyl }}-\mathrm{H}$, whereas for backbone dihedral angles we considered four consecutive backbone carbon atoms. As shown in Table IV, transitions between conformational states of methyl groups are observed at each temperature and all the dihedral angles are active in the whole temperature range. On the contrary, in the case of backbone
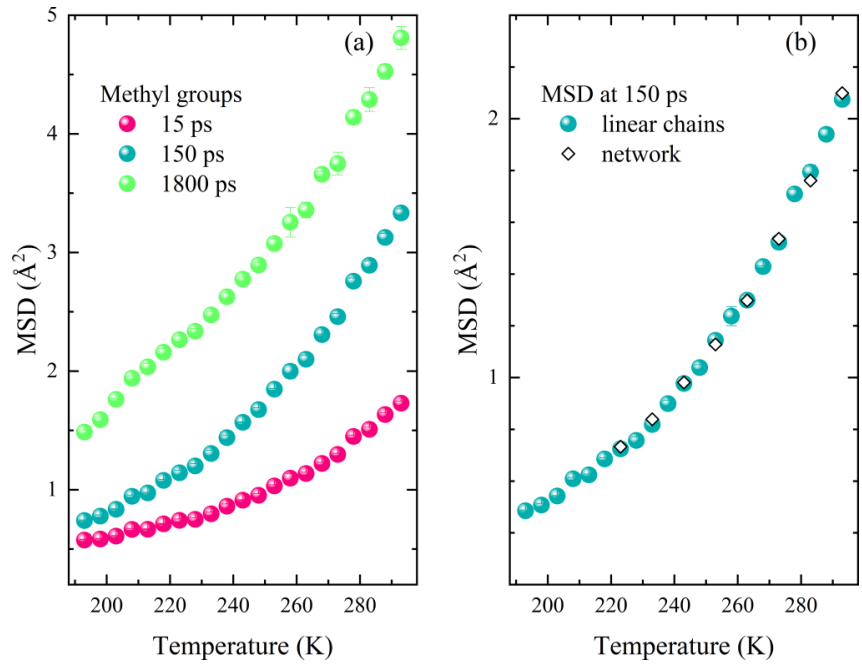

FIG. 12. (a) Temperature dependence of MSDs of hydrogen atoms belonging to the methyl groups calculated at different time resolution: 15 ps (pink), 150 ps (blue), and 1800 ps (green). (b) Comparison between the temperature dependence of PNIPAM hydrogen atom MSDs calculated from MD simulations at 150 ps at a concentration of $60 \mathrm{wt} . \%$ of linear polymer chains (blue circles) or polymer network (white diamonds) [17]. When not visible, error bars are within symbol size.

dihedral angles, a clear increase of the number of mobile dihedral angles occurs at $T_{d}$.

We quantitatively compared the experimental and numerical results by calculating the numerical MSD of PNIPAM hydrogen atoms from the equation

$$
\operatorname{MSD}(t)=\left\langle\left|r_{\mathrm{H}}(t)-r_{\mathrm{H}}(0)\right|^{2}\right\rangle,
$$

where $r_{\mathrm{H}}(t)$ and $r_{\mathrm{H}}(0)$ are the position vectors of a PNIPAM hydrogen atom at time $t$ and 0 , averaged over time origins and hydrogen atoms. In addition to the MSDs of all PNIPAM hydrogen atoms and those in the backbone, we have also calculated the contribution of the MSDs of the hydrogen atoms in the methyl groups which also exhibits a change at $T_{d} \sim 225 \mathrm{~K}$ (see Fig. 12(a)).

Finally, to evaluate the role of the molecular architecture on the polymer local dynamics, we have reported in Fig. 12(b) a comparison between the temperature dependence of the MSDs of PNIPAM hydrogen atoms of linear chains and microgel networks, whose data were taken from Ref. [17]. Figure 12(b) shows quantitative agreement between the MSDs calculated at 150 ps with no differences between the two polymer architectures.
[1] W. Doster, S. Cusack, and W. Petry, Dynamical transition of myoglobin revealed by inelastic neutron scattering, Nature (London) 337, 754 (1989).

[2] K. L. Ngai, S. Capaccioli, and A. Paciaroni, Change of caged dynamics at $T_{g}$ in hydrated proteins: Trend of mean squared displacements after correcting for the methyl-group rotation contribution, J. Chem. Phys. 138, 235102 (2013).
[3] G. Schirò and M. Weik, Role of hydration water in the onset of protein structural dynamics, J. Phys.: Condens. Matter 31, 463002 (2019).

[4] G. Schirò, Y. Fichou, F.-X. Gallat, K. Wood, F. Gabel, M. Moulin, M. Härtlein, M. Heyden, J.-P. Colletier, A. Orecchini et al., Translational diffusion of hydration water correlates with functional motions in folded and intrinsically disordered proteins, Nat. Commun. 6, 6490 (2015). 
[5] K. Wood, M. Plazanet, F. Gabel, B. Kessler, D. Oesterhelt, D. Tobias, G. Zaccai, and M. Weik, Coupling of protein and hydration-water dynamics in biological membranes, Proc. Natl. Acad. Sci. USA 104, 18049 (2007).

[6] M. Ferrand, A. J. Dianoux, W. Petry, and G. Zaccai, Thermal motions and function of bacteriorhodopsin in purple membranes: Effects of temperature and hydration studied by neutron scattering, Proc. Natl. Acad. Sci. USA 90, 9668 (1993).

[7] G. Zaccai, How soft is a protein? A protein dynamics force constant measured by neutron scattering, Science 288, 1604 (2000).

[8] M. Tarek and D. J. Tobias, Role of Protein-Water Hydrogen Bond Dynamics in the Protein Dynamical Transition, Phys. Rev. Lett. 88, 138101 (2002).

[9] K. Wood, A. Frölich, A. Paciaroni, M. Moulin, M. Härtlein, G. Zaccai, D. J. Tobias, and M. Weik, Coincidence of dynamical transitions in a soluble protein and its hydration water: Direct measurements by neutron scattering and md simulations, J. Am. Chem. Soc. 130, 4586 (2008).

[10] H. Nakagawa and M. Kataoka, Percolation of hydration water as a control of protein dynamics, J. Phys. Soc. Jpn. 79, 083801 (2010).

[11] O. Rahaman, M. Kalimeri, M. Katava, A. Paciaroni, and F. Sterpone, Configurational disorder of water hydrogen-bond network at the protein dynamical transition, J. Phys. Chem. B 121, 6792 (2017).

[12] Z. Liu, J. Huang, M. Tyagi, H. O’Neill, Q. Zhang, E. Mamontov, N. Jain, Y. Wang, J. Zhang, J. C. Smith, and L. Hong, Dynamical Transition of Collective Motions in Dry Proteins, Phys. Rev. Lett. 119, 048101 (2017).

[13] E. Cornicchi, S. Capponi, M. Marconi, G. Onori, and A. Paciaroni, Temperature dependence of fast fluctuations in single- and double-stranded DNA molecules: A neutron scattering investigation, Philos. Mag. 87, 509 (2007).

[14] G. Caliskan, R. M. Briber, D. Thirumalai, V. Garcia-Sakai, S. A. Woodson, and A. P. Sokolov, Dynamic transition in tRNA is solvent induced, J. Am. Chem. Soc. 128, 32 (2006).

[15] J. Peters, J. Marion, F. Natali, E. Kats, and D. J. Bicout, The dynamical transition of lipid multilamellar bilayers as a matter of cooperativity, J. Chem. Phys. B 121, 6860 (2017).

[16] M. Zanatta, L. Tavagnacco, E. Buratti, M. Bertoldo, F. Natali, E. Chiessi, A. Orecchini, and E. Zaccarelli, Evidence of a lowtemperature dynamical transition in concentrated microgels, Sci. Adv. 4, eaat5895 (2018).

[17] L. Tavagnacco, E. Chiessi, M. Zanatta, A. Orecchini, and E. Zaccarelli, Water-polymer coupling induces a dynamical transition in microgels, J. Phys. Chem. Lett. 10, 870 (2019).

[18] A. Fernandez-Nieves, H. Wyss, J. Mattsson, and D. A. Weitz, Microgel Suspensions: Fundamentals and Applications (Wiley, New York, 2011).

[19] Y. He, P. I. Ku, J. R. Knab, J. Y. Chen, and A. G. Markelz, Protein Dynamical Transition Does Not Require Protein Structure, Phys. Rev. Lett. 101, 178103 (2008).

[20] G. Schirò, C. Caronna, F. Natali, M. M. Koza, and A. Cupane, The "protein dynamical transition" does not require the protein polypeptide chain, J. Phys. Chem. Lett. 2, 2275 (2011).

[21] J. Rubio Retama, B. Frick, T. Seydel, M. Stamm, A. Fernandez Barbero, and E. López Cabarcos, Polymer chain dynamics of core-shell thermosensitive microgels, Macromolecules 41, 4739 (2008).
[22] M. Karg, A. Pich, T. Hellweg, T. Hoare, L. A. Lyon, J. J. Crassous, D. Suzuki, R. A. Gumerov, S. Schneider, I. I. Potemkin et al., Nanogels and microgels: From model colloids to applications, recent developments, and future trends, Langmuir 35, 6231 (2019).

[23] L. Rovigatti, N. Gnan, L. Tavagnacco, A. J. Moreno, and E. Zaccarelli, Numerical modeling of non-ionic microgels: An overview, Soft Matter 15, 1108 (2019).

[24] S. Fujishige, K. Kubota, and I. Ando, Phase transition of aqueous solutions of poly( $N$-isopropylacrylamide) and poly( $N$-isopropylmethacrylamide), J. Phys. Chem. 93, 3311 (1989).

[25] E. I. Tiktopulo, V. N. Uversky, V. B. Lushchik, S. I. Klenin, V. E. Bychkova, and O. B. Ptitsyn, "Domain" coil-globule transition in homopolymers, Macromolecules 28, 7519 (1995).

[26] F. Afroze, E. Nies, and H. Berghmans, Phase transitions in the system $\operatorname{poly}(N$-isopropylacrylamide)/water and swelling behavior of the corresponding networks, J. Mol. Struct. 554, 55 (2000).

[27] www.ill.eu/users/instruments/.

[28] M. Zanatta, M. Bertoldo, E. Buratti, F. Natali, J. Ollivier, A. Orecchini, J. M. Ruiz Franco, L. Tavagnacco, and E. Zaccarelli, Low-temperature dynamical transition in polymeric aqueous environments, Institut Laue-Langevin, 2018, doi:10.5291/ILLDATA.9-11-1866.

[29] L. Tavagnacco, M. Bertoldo, E. Buratti, F. Camerin, B. Frick, J. Ollivier, A. Orecchini, P. Tozzi, E. Zaccarelli, and M. Zanatta, Investigation of Supercooled Water Dynamics by Confinement in Dense Microgel Suspensions, Institut Laue-Langevin, 2018, doi:10.5291/ILL-DATA.9-11-1864.

[30] L. Tavagnacco, E. Zaccarelli, and E. Chiessi, On the molecular origin of the cooperative coil-to-globule transition of $\operatorname{poly}(\mathrm{N}$ isopropylacrylamide) in water, Phys. Chem. Chem. Phys. 20, 9997 (2018).

[31] S. Páll, M. J. Abraham, C. Kutzner, B. Hess, and E. Lindahl, in Solving Software Challenges for Exascale: International Conference on Exascale Applications and Software, Stockholm, 2014, Revised Selected Papers, edited by S. Markidis and E. Laure (Springer International, Cham, 2015), pp. 3-27.

[32] M. J. Abraham, T. Murtola, R. Schulz, S. Páll, J. C. Smith, B. Hess, and E. Lindahl, GROMACS: High performance molecular simulations through multi-level parallelism from laptops to supercomputers, SoftwareX 1-2, 19 (2015).

[33] W. L. Jorgensen, D. S. Maxwell, and J. Tirado-Rives, Development and testing of the OPLS all-atom force field on conformational energetics and properties of organic liquids, J. Am. Chem. Soc. 118, 11225 (1996).

[34] S. W. I. Siu, K. Pluhackova, and R. A. Böckmann, Optimization of the OPLS-AA force field for long hydrocarbons, J. Chem. Theory Comput. 8, 1459 (2012).

[35] J. L. F. Abascal, E. Sanz, R. G. Fernández, and C. Vega, A potential model for the study of ices and amorphous water: TIP4P/Ice, J. Chem. Phys. 122, 234511 (2005).

[36] G. Bussi, D. Donadio, and M. Parrinello, Canonical sampling through velocity rescaling, J. Chem. Phys. 126, 014101 (2007).

[37] W. Humphrey, A. Dalke, K. Schulten et al., VMD: Visual molecular dynamics, J. Mol. Graph. 14, 33 (1996).

[38] G. Camisasca, M. De Marzio, D. Corradini, and P. Gallo, Two structural relaxations in protein hydration water and their dynamic crossovers, J. Chem. Phys. 145, 044503 (2016). 
[39] J. H. Roh, V. N. Novikov, R. B. Gregory, J. E. Curtis, Z. Chowdhuri, and A. P. Sokolov, Onsets of Anharmonicity in Protein Dynamics, Phys. Rev. Lett. 95, 038101 (2005).

[40] G. Schirò, C. Caronna, F. Natali, and A. Cupane, Direct evidence of the amino acid side chain and backbone contributions to protein anharmonicity, J. Am. Chem. Soc. 132, 1371 (2010).

[41] G. Schirò, F. Natali, and A. Cupane, Physical Origin of Anharmonic Dynamics in Proteins: New Insights from ResolutionDependent Neutron Scattering on Homomeric Polypeptides, Phys. Rev. Lett. 109, 128102 (2012).

[42] M. Katava, G. Stirnemann, M. Zanatta, S. Capaccioli, M. Pachetti, K. Ngai, F. Sterpone, and A. Paciaroni, Critical structural fluctuations of proteins upon thermal unfolding challenge the Lindemann criterion, Proc. Natl. Acad. Sci. USA 114, 9361 (2017).

[43] B. F. Rasmussen, A. M. Stock, D. Ringe, and G. A. Petsko, Crystalline ribonuclease a loses function below the dynamical transition at $220 \mathrm{~K}$, Nature (London) 357, 423 (1992).

[44] A. Iorio, G. Camisasca, and P. Gallo, Slow dynamics of hydration water and the trehalose dynamical transition, J. Mol. Liq. 282, 617 (2019).

[45] Neutron Data Booklet, edited by A. Dianoux and G. Lander (Institute Laue Langevin, Grenoble, 2002).

[46] H. W. Weber and R. Kimmich, Anomalous segment diffusion in polymers and NMR relaxation spectroscopy, Macromolecules 26, 2597 (1993). 